East African Medical Journal Vol. 81 No. 2 February 2004

MANAGING SCALP DEFECTS IN SUB-SAHARAN AFRICA

J. N. Legbo, FWACS, FMCS, FRCSEd, FICS, Plastic and Reconstructive Surgery and B.B. Shehu, FRCSI, FWACS, Neurosurgery unit, Department of Surgery,

Usmanu Danfodiyo University Teaching Hospital, P.M.B. 2370, Sokoto, 840001, Nigeria

Request for reprints to: Dr. J. N. Legbo, Plastic and Reconstructive Surgery Unit, Department of Surgery, Usmanu Danfodiyo University Teaching Hospital, P.M.B. 2370, Sokoto, 840001 , Nigeria

\title{
MANAGING SCALP DEFECTS IN SUB-SAHARAN AFRICA
}

\author{
J. N. LEGBO and B.B. SHEHU
}

\begin{abstract}
Objectives: To determine the common aetiological factors of scalp defects, and outcome of management.

Design: A two year prospective study.

Setting: Usmanu Danfodiyo University Teaching Hospital, Sokoto, Nigeria.

Subjects: All consecutive patients with scalp defect from January 2001 to December 2002.

Main outcome measures: Size of defect, associated bone loss, osteomyelitis, type of surgery, duration of hospital stay and complications of surgery.

Interventions: Patients went through a simple management protocol involving history, clinical examination, relevant tests and appropriate treatment, including surgery.

Results: A total of 27 patients were studied out of which 15 were males and 12 females, giving a male to female ratio of 1.25: 1 . The age range was seven months - 42 years (mean = 13.9 years). Road Traffic Accidents (RTAs) was the commonest cause of scalp defects $(\mathbf{8 1 . 5 \%})$. The temporo-parietal area was involved in over $50 \%$ of patients. Chronicity and osteomyelitis were common complications of the defects. Over $50 \%$ of the patients had local flap reconstruction.

Conclusions: Management of scalp defects remains a major challenge in our environment. The importance of continuing education of colleagues and other health workers in peripheral health units on the importance of proper initial wound debridement and early referral cannot be overemphasised.
\end{abstract}

\section{INTRODUCTION}

Numerous reports, beginning with the Egyptians in 3000B.C. and extending to efforts during the Industrial Revolution, attest to the difficulties in managing scalp defects(1). Many of these unfortunate patients were doomed to die because of prolonged infections and intracranial complications. Those who survived had chronic wounds, with denuded bones, sequestration and dense scar. These chronic ulcers often terminated in scar carcinoma. The basic principle therefore, when approaching defects of scalp, calvarium and forehead is early soft tissue coverage of exposed bones. Soft tissue coverage alone over a bony defect does not provide protective covering of intracranial contents and is often lacking in proper contour. However, bony reconstruction to restore contour and skull integrity remains secondary to adequate wound closure.

The scalp is a peculiar anatomical entity in that it is thick, hair bearing, endowed with a rich blood supply and therefore offers varying opportunities to the reconstructive surgeon. Avulsion injuries commonly produce partial thickness losses in the subgaleal layer(1). Small avulsion injuries of that nature can be managed by primary closure in a fashion similar to that for simple scalp lacerations; that is, after undermining and galeal scoring. Although many options exist for providing initial wound cover of large avulsion losses with intact pericranium, skin grafting is generally favoured. Secondary scalp reconstruction can then proceed after initial wound healing.

Full thickness defects, involving loss of all layers, including pericranium, are more difficult to manage in the sense that skin graft will not survive on bare bone. Furthermore, exposed calvarium deprived of its periosteal blood supply is prone to necrosis and sequestration. This therefore calls for a more aggressive approach to primary wound closure(2). The technique used may vary with cause and size of the defect and the quality of the surrounding local tissue available for use. Such techniques include outer table removal with direct or delayed grafting; pericranial flap and skin graft; local flap(s) with galeal scoring; distant or microvascular flap transfer; and tissue expansion(1).

Over the years in developing countries, patients with scalp defects are initially seen and managed at peripheral health units before referral to specialist reconstructive and /or neurosurgical centers(3). The problems encountered by such referrals have been blamed on a mirage of factors including poor facilities 
at the peripheral health units, ignorance on the part of the personnel and lack of funds on the part of the patients and their relations (3). This ultimately translates to poor initial wound care, delayed referrals and unsatisfactory outcome. This study is a report of experience with managing scalp defects in tropical Africa.

\section{MATERIALS AND METHODS}

This two year prospective study was conducted between January 2001 and December 2002 at the Usmanu Danfodiyo University Teaching Hospital, Sokoto, Nigeria. The hospital serves the health needs of all the states in the North Western part of Nigeria, with a population well over 20 million people. All consecutive patients seen with scalp defects were included in the study. Each patient's information was entered into a study proforma and subsequently transferred into the computer for analysis. Patients were thoroughly evaluated at initial assessment to determine the exact nature of scalp defect and ascertain the involvement or otherwise of other system(s).

This included a detailed clinical evaluation, clinical photography, relevant microbiological, haematological, chemical, histological and radiological investigations. patients were resuscitated and wound care measures instituted where necessary. Tetanus toxoid (if not previously given) and antibiotics were administered. Definitive surgery was anchored on the variables that each patient presented with. All patients were followed-up after discharge from hospitalisation at the surgical out patient unit.

\section{RESULTS}

There were a total of 27 patients out of which there were 15 males and 12 females giving a male to female ratio of 1.25: 1. The age range was seven months 42 years $($ mean $=13.9$ years $)$. Figure 1 shows the age/ sex distribution of patients. Road Traffic Accidents (RTAs) was the commonest cause of scalp defect as seen in 22(81.5\%) patients. This was followed by burns and tumour ablation in two $(7.4 \%)$ patients each and scalp infection (necrotising fascitis) in one $(3.7 \%$ ) patient. Of the 22 patients whose defects resulted from RTAs, 13(59.0\%) were from motorcycle accidents.

The duration of defect before first hospital treatment was sought in the 24 patients with RTAs and burns was 30 minutes -6 hours (mean $=2.5$ hours). However, in $21(77.8 \%)$ patients who were referred to our unit (not first seen by us after the defect was sustained), the duration before referral was five days three months (mean $=5$ weeks). In some patients, the defect extended across anatomical regions of the scalp. However, the temporal region was the commonest site of defect as this was seen in $21(77.8 \%)$ patients. This was followed by the parietal region in $16(59.3 \%)$, the frontal region in nine $(33.3 \%)$ and the post- auricular region in two $(7.4 \%)$ patients. The size of the defect ranged from $25.0 \mathrm{~cm}^{2}$ to $96.0 \mathrm{~cm}^{2}$, with a mean of $64.3 \mathrm{~cm}^{2}$. Skull was exposed (without pericranial cover) in 19(70.4\%) patients. The exposed surface bone area ranged from
$15.2 \mathrm{~cm}^{2}$ to $80.0 \mathrm{~cm}^{2}$ with a mean of $47.0 \mathrm{~cm}^{2}$. Skull defect (bone loss) was observed in $11(40.7 \%$ ) patients. The area of such bone loss ranged from $14.6 \mathrm{~cm}^{2}$ to $60.7 \mathrm{~cm}^{2}$, with a mean of $37.5 \mathrm{~cm}^{2}$. Of the 11 patients with skull defect, seven were from sequestration, three from direct trauma resulting in fracture and one followed tumour ablation. Of the 22 patients involved in RTAs, seven lost consciousness at the time of incident, two each had long bone fracture and several stones in the scalp wound, and three others had various injuries involving the eyelid and hand.

Figure 1

\section{Age/Sex distribution}

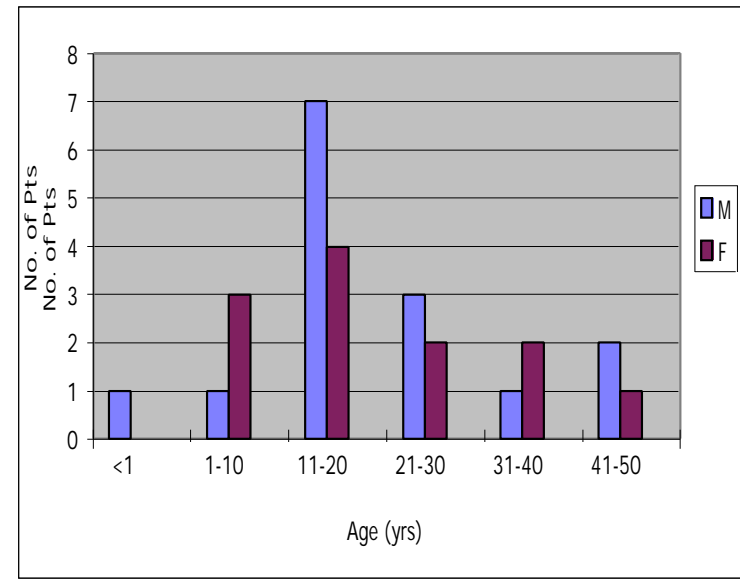

Figure 2a

A two year old girl with full thickness right parietal scalp defect

Note: Central necrosis of the pericranium due to delayed presentation 
Figure 2b

Same patient in figure $2 a$ during rotation and transposition flap surgery

Figure 2c

Same patient in figure 2 a following Galeal scoring (Transverse incisions) on the flap to allow tension-free closure

Figure 2d

Same patient in figure $2 a$ during the first wound inspection five days after surgery
Figure 2e

Same patient in figure $2 a$ at the first out patient followup visit four weeks after surgery

Twenty three $(85.2 \%)$ patients needed wound debridement. Antibiotics were given according to sensitivity results in all cases of RTAs and those who showed evidence of wound infection. Sixteen (59.3\%) patients had local flap wound cover while the remaining $11(40.7 \%)$ had split thickness skin grafting. Blood was transfused in nine $(33.3 \%)$ patients. The volume of blood transfused ranged from $1.20 \mathrm{mls}$ to $1.500 \mathrm{mls}$, with mean of $637.4 \mathrm{mls}$. Post operative morbidity was minimal: three patients had partial skin graft loss (from infection and graft shift) but did well on regular dressing and required no further grafting, one patient had partial flap necrosis which necessitated split thickness skin grafting; while another patient had persistent discharging sinus that resolved after curettage. No mortality was recorded during the period of study and follow up. The duration of hospital stay ranged from 15 days to seven weeks, with a mean of 3.5 weeks. Follow up was generally impressive and ranged from six weeks to 18 months (mean $=7$ months).

\section{DISCUSSION}

The management of scalp defects remains a continuous challenge to the reconstructive surgeon worldwide(1-3). This becomes even so in many centers in developing countries where presentation and dearth of personnel and equipment's remains the norm. Though in this study, the total number of patients seen appears relatively small, this compares well with figures from other reports(3). Figure 1 confirms the fact that no age or sex is immune to this ever challenging reconstructive surgical problem. Most of the patients belong to the teenage age group and those aged 30 years and below. This is not surprising as people in this age bracket are quite active and mobile, predisposing them to all manners of accidents; road traffic, industrial and/or domestic. 
The manufacturing and use of high speed automobiles, coupled with poor road/vehicular maintenance, and disobedience to traffic rules may have accounted for road traffic accidents as seen in $81.5 \%$ of patients in our series. The use of motorcycles remains an important mode of transportation in both rural and urban settlements in Nigeria as in most developing countries. Most of the cyclists (commercial and private) do not use protective crash helmets as no law in the country necessitated the use of such during the period of this study. A good number of the victims of such motorcycle accidents are the passengers who, with little or no support, loose grip of the vehicle and commonly land on the head in the event of an accident. This may explain why $13(59.0 \%)$ of the 22 patients involved in road traffic accidents (RTAs) resulted from motorcycle accidents. Scalp infection (necrotising fascitis) from prolonged intravenous canulation could also result in scalp loss as was seen in one patient in this study. Similarly, scalp arterial canulation could result in ischaemic scalp necrosis.

The mean duration of defect of 2.5 hours before first hospital treatment was sought by patients with RTAs and burns is rather short compared to the general presentation of surgical patients in our centre. This could be a result of the dramatic nature of scalp injuries that may be attended by bleeding or pain as the case may be. Unfortunately, it took an average of five weeks before most patients were referred for wound cover. The reasons for such delays included failure of recognition of the need for prompt soft tissue coverage of full thickness defects; unwillingness by patients and their relations to accept such transfers; lack of funds by patients; and time needed to stabilise some of the patients with other injuries. Referrals within other units in our hospital came within hours to a few days as compared to those from other centres, which came as late as three months after the injury was sustained. The sites of the defects is probably a reflection of the mechanism of injuries that led the defect; the two patients with post auricular defects followed surgical ablation of an osteoma of the skull and oncocytoma of the parotid gland.

Exposure of skull (skull without pericranial cover) strips it of its supply of blood and nutrients, thereby predisposing it to necrosis and sequestration as was observed in seven of the patients in our study. This is largely preventable by various techniques(3) that minimise the duration of exposure as highlighted in the introduction. Wound debridement was necessary in a good number of patients, even in most of whom such procedures had been carried out before referral to our unit. In two of such patients, several foreign bodies including stones were extracted from the wound to gain control of the ongoing osteomyelitis and prepare the wound for cover. All except two of the 23 patients who had local flap cover had rotational flaps of the scalp. This was possible since most of the defects were located at the temporoparietal region, allowing for a wide are though the contralateral side of the head. Due to non-availability of Doppler in our center, as in most other centers in the developing countries, all such flaps were based on named arteries, the identification of which were mainly on digital palpation and by the understanding of their anatomical surface markings. Galeal scoring of the flap and undermining of the adjacent scalp were done in all defects to aid closure without tension, criss crossing galeal incisions were also made where additional flap width was required. These gave us the advantage of closing the secondary defect directly in all the patients. This is one major advantage of rotational as opposed to transposition flaps(1). Such secondary defects created after transposition flaps usually require glabrous skin graft. Such glabrous skin graft has the disadvantages of being insensate, of poor quality (unstable), hairless and therefore requires secondary flap reconstruction by tissue expansion(1,3,5). Galeal scoring needs to be done with a great deal of care, as any damage to the vessels in the supragaleal plane may be heralded by flap necrosis. Advancement flaps can be used to full thickness scalp defects. Large areas of the posterior scalp may be closed by this method, taking advantage of loose neck skin and neck extension as was the case in two of our patients who had post auricular defects following tumour ablation. The main problem with this flap design is with its limited ability to move. In addition, advancement of anterior scalp to cover any defect might raise the brows to an objectional height.

The pericranium is similar to periosteum of long bones except that it is thicker especially in young patients. Endowed with a rich vascular supply, it may be utilized as a pedicle flap that provides coverage of denuded bone and act as a bed for skin grafting $(1,4)$. It can also be used as a vascular covering over areas of bone grafting for calvarial reconstruction to enhance graft survival. The great advantage of pericranial flaps is their minimal donor site morbidity and their dependability as a bed for skin grafting if properly designed. For no particular reason however, we did not use this flap in any of our patients in this series. Eleven $(40.7 \%)$ patients had split thickness skin grafting (STSG) either because their wounds were already covered with granulation tissue at the time of referral to our unit, or such granulation tissue covered exposed bone while the patient was waiting for funds for surgery. One patient had STSG because the wound was too large to allow any meaningful flap reconstruction. All these patients are to undergo secondary reconstruction after skin expansion to improve the aesthetic appearance.

Skull defects in the parietal and occipital areas may represent less of a problem from a cosmetic point of view but may still require cranioplasty for protective purposes. Defects in the temporal region are less obvious, being somewhat protected by the overlying temporalis muscle, and thus less frequently require 
cranioplasty. Defects in the frontal region warrant cranioplasty for both cosmetic and protective reasons. In general, however, defects larger than $10 \mathrm{~cm}^{2}$ are reconstructed(3). The timing of reconstruction depends on many factors including the condition of the patient, associated injuries, and the size and nature of the underlying defect. Numerous alloplastic materials (vitallium, tantalum, stainless steel, acrylic, etc.) have been used to restore bony skull defects(4). For most plastic surgeons, autogenous bone remains the material of choice for reconstructing calvarial defects. The advantages of reconstructing with like tissue are many, although, most important, the use of autogenous bone is associated with a low incidence of infection and graft loss. In our study, nearly half of the 11 patients with bone loss will require calvarial reconstruction.

Although post operative morbidity was minimal, the duration of hospital stay was unacceptably prolonged in many patients due to delays in referral and poor initial wound care. This is in sharp contrast with certain reports where emergency repair of the scalp was carried out with intraoperative skin expansion(2). In conclusion that scalp defects remain an important cause of morbidity in our environment. The dearth of facilities, infrastructures and manpower, and delay in referral to specialist centers all militate against the effective treatment of this condition in the developing world. Maintenance of roads/automobiles and obedience to traffic rules and regulations will reduce the incidence of such defects resulting from trauma. Furthermore, the importance of the use of protective crash helmets by motorcyclists cannot be overemphasised. Adequate initial wound debridement and prompt referral will reduce the morbidity and mortality attended to such injuries.

\section{REFERENCES}

1. Freund, R.M. Scalp, calvarium and forehead reconstruction. In: Grab and Smith's Plastic Surgery, 5th edition. Edited by Aston S.J., Beasley R.W. and Thorne C.H.M. LippincottRaven Publishers, Philadelphia. 1997; 473-482.

2. Pianigiani, E., Andreassi, A., Biagioli, M., Rubegni, P., Castelli, A. and Taddeucci, P. Intraoperative skin expansion in emergency repair of the scalp. J. Derm. Treat. 2001; 12:25-28.

3. Jurkiewiez, M. J. and Hill, H. L. Open wounds of the scalp: an account of methods of repair. J. Trauma. 1981; 21:769778.

4. McGregor I. A. Fundamental Techniques of Plastic Surgery, 8th edition. Churchill Livingstone. 1990; 119-124.

5. Argenta, L. C., Watanabe M. J. and Grabb ,W.C. The use of tissue expansion in head and neck reconstruction. Ann. Plast. Surg. 1983; 11:31-37. 